\title{
Les amyloses héréditaires
}

Les amyloses sont des maladies caractérisées par le dépôt de protéines dénaturées, insolubles, dans les tissus. Leur symptomatologie dépend de l'importance et des localisations tissulaires de ces dépôts. Des mutations ponctuelles de protéines circulantes peuvent être à l'origine d'amyloses héréditaires, qui sont alors toujours des maladies dominantes. Parmi les plus fréquentes des protéines ainsi altérées, on trouve la transthyrétine, la gelsoline et l'apolipoprotéine A1. Le tableau clinique de ces affections varie avec la protéine mutée, la nature de la mutation et parfois même chez des malades de familles différentes portant la même mutation, ce qui suggère que $d$ 'autres caractéristiques génétiques interviennent dans le processus amyloïdogénique. L'expression, chez des souris transgéniques, d'une transthyrétine mutée amyloïdogénique permet de reproduire certaines des lésions anatomiques observées chez l'homme et pourrait donc constituer un intéressant modèle pour l'étude physiopathologique et thérapeutique de cette maladie.

\section{Gilles Grateau}

\section{ADRESSE}

G. Grateau : praticien hospitalier universitaire. Service de médecine interne, hôpital Cochin, URA 1147 Cnrs, Institut Cochin de génétique moléculaire (ICGM), 27, rue du Faubourg-Saint-Jacques, 75674 Paris es amyloses sont un ensemble de maladies caractérisées par le dépôt, presque toujours extracellulaire, de protéines ayant une conformation fibrillaire [1]. Ces dépôts se font dans de nombreux tissus et sont responsables de manifestations cliniques très polymorphes et le plus souvent graves. Pendant longtemps la connaissance des amyloses est restée anatomoclinique et ce n'est que dans les années 1970 que les progrès de la biochimie ont permis une analyse précise de la substance amyloïde. Il est alors apparu qu'elle était composée pour $85 \%$ d'une protéine amyloïde de structure fibrillaire, spécifique de chaque variété d'amylose, et pour $15 \%$ de plusieurs protéines et glycoprotéines communes à toutes les variétés. Une quinzaine de protéines amyloïdes ont été depuis découvertes, ce qui a permis d'élaborer une classification biochimico-clinique [2] (Tableau I).

Les formes familiales d'amylose ont été reconnues depuis longtemps et recouvrent en fait des entités différentes : (1) des maladies héréditaires dont le gène est connu et où la mutation porte sur la protéine amyloïde elle-même ; (2) des maladies héréditaires dont le gène n'est pas connu et où la place de l'amylose, complication ou association, n'est pas parfaitement définie, ce qui est le cas de la maladie périodique; (3) la présence de plusieurs cas d'amylose dans une même famille, où la nature héréditaire de la maladie n'est pas prouvée. Nous ne traiterons ici que des 
amyloses héréditaires bien définies [2], en nous limitant à celles qui sont les mieux connues et en excluant les amyloses du système nerveux central (Tableau II, p. 526).

\section{Historique}

La découverte des amyloses héréditaires est l'œuvre d'Andrade au Portugal [4], bien que quelques observations de neuropathies héréditaires, probablement amyloïdes, aient été décrites antérieurement [5]. La transmission génétique se fait toujours sur le mode autosomique dominant. Le nerf périphérique est l'organe cible principal, mais d'autres peuvent être touchés, et une première classification des neuropathies amyloïdes familiales a ainsi été élaborée en fonction des caractéristiques cliniques de la neuropathie et de la nature de l'atteinte extra-neurologique. En fait, on sait maintenant qu'une même protéine amyloïde pcut être associée à différentes formes cliniques, et qu'inversement des protéines amyloïdes différentes peuvent donner des manifestations cliniques identiques. Nous présenterons donc les amyloses héréditaires en suivant pour l'essentiel une classification biochimique, en développant les amyloses de la transthyrétine (TTR) qui sont les plus fréquentes et les mieux connues.

\section{Les amyloses de la transthyrétine}

Les neuropathies périphériques des amyloses de la TTR sont initialement sensitives et douloureuses, puis motrices et comportent le plus souvent une atteinte du système nerveux autonome avec hypotension orthostatique, impuissance et troubles du transit intestinal. L'évolution est sévère et conduit à un état grabataire en 10 à 15 ans. Les dépôts vitréens surviennent le plus souvent au cours de l'évolution de la maladie, mais peuvent précéder la neuropathie, parfois de plusieurs années. L'atteinte cardiaque entraîne une insuffisance cardiaque avec troubles du rythme ou de la conduction, d'évolution subaiguë. Le rein et le tube digestif sont les autres organes dont l'envahissement par l'amylose a une répercussion clinique importante. Le diagnostic de la $\mathrm{m} / \mathrm{s} \mathrm{n}^{\circ} 6$, vol. 8, juin-juillet 92

Tableau I

NOMENCLATURE ET CLASSIFICATION DES AMYLOSES HUMAINES (D'après [2])

\begin{tabular}{|c|c|c|}
\hline Protéine amyloïde & Précurseur & Amylose \\
\hline AA & $\operatorname{apoSAA}{ }^{*}$ & $\begin{array}{l}\text { réactionnelle : } \\
\text { infections, } \\
\text { inflammations } \\
\text { chroniques } \\
\text { tumeurs } \\
\text { maladie périodique, } \\
\text { syndrome de Muckle } \\
\text { et Wells }\end{array}$ \\
\hline$A L$ & $\begin{array}{l}\text { chaîne légère d'Ig } \\
(\kappa, \lambda)\end{array}$ & $\begin{array}{l}\text { idiopathique (primitive) } \\
\text { associée au myélome } \\
\text { ou à la maladie } \\
\text { de Waldenström }\end{array}$ \\
\hline $\mathrm{AH}$ & $\begin{array}{l}\text { chaîne lourde d'lgG } \\
(\gamma)\end{array}$ & \\
\hline ATTR & $\begin{array}{l}\text { transthyrétine mutée } \\
\text { transthyrétine normale }\end{array}$ & $\begin{array}{l}\text { héréditaire } \\
\text { sénile }\end{array}$ \\
\hline AАpoA 1 & apoA1 & $\begin{array}{l}\text { neuropathie } \\
\text { héréditaire }\end{array}$ \\
\hline AGel & gelsoline & $\begin{array}{l}\text { neuropathie } \\
\text { héréditaire }\end{array}$ \\
\hline ACys & cystatine C & $\begin{array}{l}\text { hémorragie cérébrale } \\
\text { héréditaire }\end{array}$ \\
\hline $\mathrm{A} \beta$ & $\begin{array}{l}\text { précurseur } \\
\text { de la protéine } \beta \\
\text { App }\end{array}$ & $\begin{array}{l}\text { maladie d'Alzheimer } \\
\text { trisomie } 21 \\
\text { hémorragie cérébrale } \\
\text { héréditaire }\end{array}$ \\
\hline $\mathrm{A} \beta 2 \mathrm{M}$ & $\beta 2$-microglobuline & $\begin{array}{l}\text { associée à l'insuffi- } \\
\text { sance rénale } \\
\text { chronique terminale }\end{array}$ \\
\hline AScr & $\begin{array}{l}\text { précurseur de la } \\
\text { protéine } \\
\text { scrapie }\end{array}$ & $\begin{array}{l}\text { encéphalopathie } \\
\text { spongiforme } \\
\text { Gerstmann-Straüssler- } \\
\text { Scheinker }\end{array}$ \\
\hline ACal & procalcitonine & $\begin{array}{l}\text { associée au cancer } \\
\text { médullaire } \\
\text { de la thyroïde }\end{array}$ \\
\hline AANF & $\begin{array}{l}\text { facteur atrial } \\
\text { natriurétique }\end{array}$ & $\begin{array}{l}\text { amylose auriculaire } \\
\text { isolée }\end{array}$ \\
\hline AIAPP & $\begin{array}{l}\text { polypeptide amyloïde } \\
\text { des îlots }\end{array}$ & $\begin{array}{l}\text { îlots de Langerhans } \\
\text { des diabètes de } \\
\text { type 2, insulinome }\end{array}$ \\
\hline
\end{tabular}

apoSSA : apo serum amyloid $A$.

maladie comporte deux étapes : (1) mise en évidence de l'amylose au sein du tissu lésé, le plus souvent par biopsie neuromusculaire, plus rarement rénale, cutanée ou cardiaque ; (2) caractérisation immunochimique de l'amylose avec des anticorps antitransthyrétine (figure $1, p .527$ ). En effet, la protéine qui a été purifiée à partir des dépôts d'amylose de ces malades est la transthyrétine, entière ou fragmentée [6]. Comme pour tou- tes les amyloses systémiques, la protéine présente dans le dépôt provient du plasma où sa concentration sérique est de $300 \mathrm{mg} / \mathrm{l}$. La dénomination TTR fait référence à ses capacités fonctionnelles de fixation de la thyroxine et de la protéine porteuse de la vitamine $\mathrm{A}$ ou retinol binding protein (RBP). Le terme de préalbumine, donné initialement à cette protéine car elle migre plus vite que l'albumine au cours d'une électrophorèse 


\section{RÉFÉRENCES}

1. Stonc MJ. Amyloidosis : a final common pathway for protein deposition in tissues. Blood 1990; 75 : 531-45

2. Husby G, Araki S, Benditt EP, et al. The 1990 guidelines for nomenclature and classification of amyloid and Amyloidosis In : Natvig JB, Forre (), Husby G, et al., cds. Amyloid and amyloidosis. Dordrecht : Kluwer Academic Publishers, 1991: 7-11.

3. Benson Ml), Wallace MR. Amyloidosis. In: Beaudet AI, Scriver CR, Sly WS, Vallc ISS, cds. Metabolic Basis of Inherited Disease. Ncw York: McGraw Hill, 1989 2439-60.

4. Andrade C. A peculiar form of peripheral neuropathy. Familial atypical generalized amyloidosis with special involvement of peripheral nerves. Brain 1952 ; 75 : 408-26

5. De Bruyn RS, Stern RO. A case of the progressive hypertrophic polyncuritis of 1)ejerine and Sottas with pathological examination. Brain 1929; 52 : 84-107.

6. Costa PP, Figucra AS, Bravo FR. Amy loid libril protcin related to prealbumin in familial amyloidotic polyncuropathy. Proc Natl Acad Sci USA 1978; 75 : 4499-503.

7. Saraiva MJM, Birken S, Costa PP Goodman 1)S. Amyloid libril protein in familial amyloidotic polyncuropathy. Portuguese type. 1)efinition of molecular abnormality in transthyretin (prealbumin). / Clin Invest 1984 ; 74 : 104-19.

8. Sasaki H, Yoshioka N, Takagi Y, Sakaki Y. Structure of the chromosomal gene for human scrum prealbumin. Gene $1985 ; 37$ 191-7

9. Almcida MR, Iongo Alves I, Sakaki Y Costa PP, Saraiva MJM. Prenatal diagnosis of familial amyloidotic neuropathy : cvidence for an carly expression of the associated transthyretin methionine 30 . Hum Genet 1990 ; 85 : 623-6.

10. Yoshioka K, Furuya H, Sasaki H Saraiva MJM, Costa PP, Sakaki Y Haplotype analysis of familial amyloidotic ncuropathy. Hum Genet $1989 ; 82$ : 9-13.

11. Saraiva MJM, Costa PP, De Witt SG Genctic expression of a transthyretin mutation in typical and late-onset Portuguesc familics with familial amyloidotic polyncuropathy. Neurology 1986 ; 36 : 1413-7.

12. Sandgren (), Holmgren G, I undgren E. Vitreous amyloidosis associated with homozygosity for the transthyretin methionine-30 gene. Arch Ophtalmol 1990 ; 108 : 1584-6.

13. Saraiva MJM, Almcida MR, Costa PP Altland K, Gawinowicz MA. Molecular analysis of an acidic transthyrctin Asn 90 variant. Am J Hum Genet 1991; 48 : 1004-8. 14. I, S, Sobcll JL, Sommer SS. From molecular variant to discase : initial steps in cvaluating the association or transthyretin M 119 with discase. Am J Hum Genet 1991 ; $50: 29-41$

15. Moses AC, Rosen HN, Moller I)E, et al. A point mutation in transthyretin increases affinity for thyroxinc and produces cuthyroid hypcrthyroxincmia. I Clin Invest $1990 ; 86: 2025-33$ des protides sériques, n'est plus utilisé actucllement. La TTR est synthétisée essentiellement par le foic, clle comporte 127 acides aminés et son poids moléculaire est $14 \mathrm{kDa}$. Elle circule normalement dans le plasma sous forme de tétramères de $56 \mathrm{kDa}$ associés de façon non covalente. Chaque monomère a spontanément une structure $\beta$ plissée qui le prédispose à former les fibrilles amyloïdes [3].

Hétérogénéité génétique des amyloses de la transthyrétine

La TTR présente dans les dépôts amyloïdes des malades portugais décrits par Andrade est qualitativement anormale : elle comporte une substitution d'un acide aminé, une valine étant remplacée par une méthionine en position 30 [7]. Cette substitution correspond à une mutation ponctuclle d'une base, une adénine remplaçant une guanine dans le gène de la TTR. Celui-ci se situe sur le chromosome 18 , en $18 \mathrm{q} 11.2$ et ne comporte qu'une seule copie de $6,9 \mathrm{~kb}$. Il est composé de quatre exons et de trois introns qui ont été entièrement séquencés : le premier exon code pour les 17 acides aminés du peptide signal et pour trois acides aminés de la protéinc mûre, le second code pour 44 acides aminés, le troisième pour 45 acides aminés et le quatrième pour 35 acides aminés [8]. L'ARN messager est retrouvé dans le foie, mais aussi dans les plexus choroïdes et l'œil. Depuis la mutation Met 30, plus de quinze mutations ponctuelles ont été décrites dans le gène de la TTR, réparties dans les exons 2, 3 et 4 (Tableau III). Le diagnostic prédictif de la maladic, chez les sujets encore asymptomatiques, est maintenant possible par la mise en évidence de certaines protéines mutées dans le plasma grâce aux techniques d'isoélectrofocalisation. Mais, surtout, les mutations peuvent être visualisées par l'utilisation de l'amplification génique in vitro ou polymerase chain reaction (PCR) et de ses dérivés : amplification suivic de coupure par une enzyme de restric-
Tableau II

AMYLOSES HÉRÉDITAIRES DONT LA MUTATION EST CONNUE

\begin{tabular}{|lll|}
\hline Gène & Mutation & \multicolumn{1}{c|}{ Syndrome } \\
\hline $\begin{array}{l}\text { Précurseur } \\
\text { de la protéine } \\
\text { amyloïde } \beta\end{array}$ & APP 717 & $\begin{array}{l}\text { Maladie d'Alzheimer à début } \\
\text { précoce }\end{array}$ \\
Cystatine C & APP 693 & $\begin{array}{l}\text { Hémorragie cérébrale héréditaire } \\
\text { de type hollandais } \\
\text { Hémorragie cérébrale héréditaire } \\
\text { de type islandais }\end{array}$ \\
Protéine scrapie & plusieurs & $\begin{array}{l}\text { Encéphalopathie spongiforme } \\
\text { Gerstmann-Straüssler-Scheinker }\end{array}$ \\
Transthyrétine & Met 30 & $\begin{array}{l}\text { Polyneuropathie amyloïde familiale } \\
\text { (Portugal, Japon) } \\
\text { Cardiopathie amyloïde familiale } \\
\text { (Danemark) }\end{array}$ \\
Apolipoprotéine & Met 111 & Formes mixtes \\
Gelsoline & Arg 26 & $\begin{array}{l}\text { Polyneuropathie amylö̈de familiale } \\
\text { (lowa) } \\
\text { Néphropathie de type Ostertag } \\
\text { Neuropathie céphalique et dystro- } \\
\text { phie grillagée de la cornée } \\
\text { (Finlande, États-Unis) }\end{array}$ \\
\hline
\end{tabular}

Les amyloses héréditaires sont hétérogènes : (1) différentes mutations dans un même gène peuvent donner des manifestations cliniques distinctes (APP et TTR) ; (2) des protéines amyloïdes distinctes peuvent donner des signes cliniques très proches (TTR et Apo A1); (3) une mutation donnée, ATTR Met 30, est associée à une grande hétérogénéité clinique. 
tion, amplification ou hybridation spécifique d'allc̀le. Un diagnostic prénatal est envisageable uniquement par étude de l'ADN car, d'une part, la TTR mutée d'origine foctale est présente à un faible taux dans le liquide amniotique et peut ne pas être détectéc, et, d'autre part, il existe un risque de contamination par la TTR mutéc maternelle quand la mère est porteuse de l'allc̀le muté [9]. Le diagnostic prénatal semble légitime dans les familles où la maladie commence tôt dans la vie et a rapidement des conséquences graves. Il est beaucoup plus discutable dans les formes à révélation tardive. A unc mutation donnéc correspond en effet une forme clinique particulière par l'âge du début, la vitesse d'évolution de la maladie, et par la nature des organes atteints. Ainsi, douze mutations sont associécs à une maladie où prédomine une neuropathic, trois sont associées à une cardiopathie prédominante (Tableau III). Cettc variabilité dans l'expression de la maladic en fonction des mutations est intrigante, et inexpliquéc.

La maladie fait intervenir d'autres facteurs que la mutation

Plus frappante encore est la variabilité clinique observée pour une mutation donnéc. Cette variabilité est maximale pour la mutation Met 30 , qui est la plus répandue des mutations de la TTR et qui pose d'abord la question de son origine. En effet, découverte au Portugal, clle a été retrouvéc dans de nombreux autres pays: Japon, Suède, Angleterre, Grèce, Italie, France, entre autres. Le fait que les Vikings aient beaucoup voyagé et que les Portugais aient fondé une colonie au Japon au XVII sic̀cle pouvait suggérer l'existence d'un fondateur unique, commun à toutes ces mutations. Des chercheurs japonais ont montré que cette hypothèse était fausse en étudiant la ségrégation familiale de différents haplotypes dans le gène TTR chez des familles portugaises et japonaises. Cette mutation siège en fait dans un point chaud de mutation (hot-spot) ct pcut être expliquée par une transition cytosine méthyléethymine [10]. Il n'y a pas, à l'heure actuclle, de résultats comparant les haplotypes portugais à ceux des autres populations curopéennes où la $\mathrm{m} / \mathrm{s} n^{\circ} 6$, vol. 8, juin-juillet 92

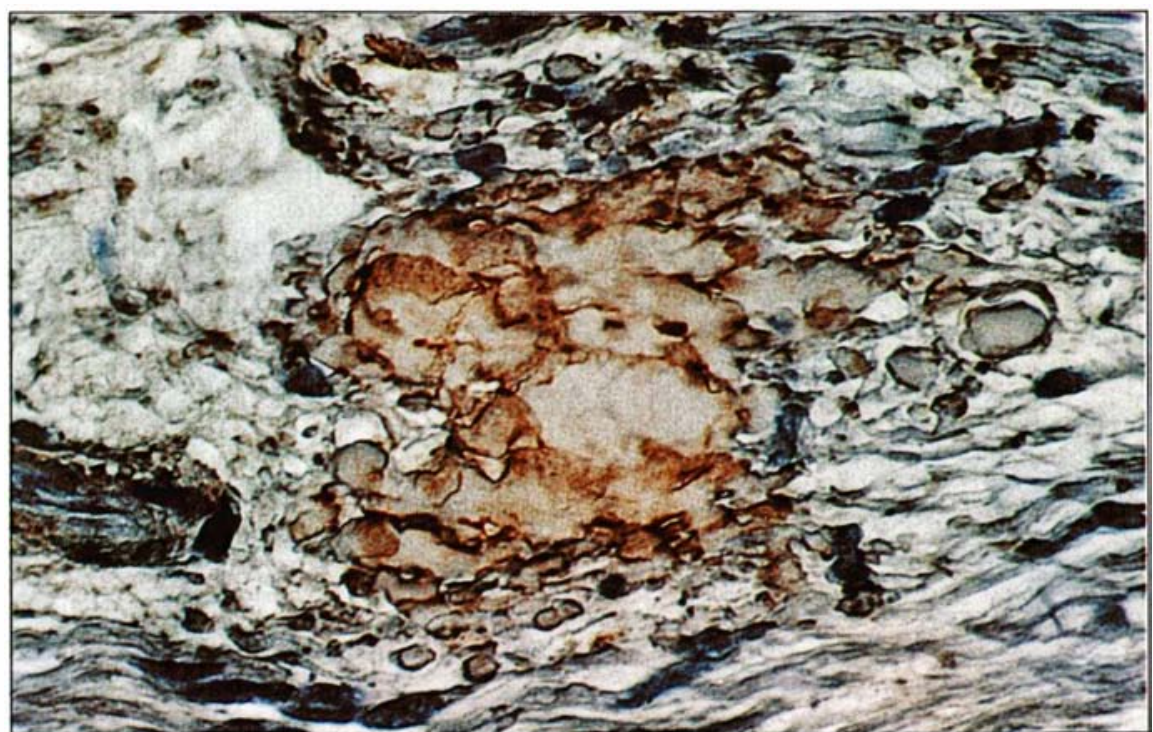

Figure 1. Étude par immunoperoxydase d'une biopsie neuromusculaire d'un malade atteint de neuropathie amyloïde héréditaire. Fixation des anticorps anti-transthyrétine (rouge) par le dépôt amyloïde (x 63). (Cliché du $\operatorname{Dr}$ C. Lacroix, laboratoire de neuropathologie du Pr. G. Said, hôpital de Bicêtre.)

Tableau III

LES AMYLOSES DE LA TRANSTHYRÉTINE

\begin{tabular}{|c|c|c|}
\hline Mutation & Signes cliniques & Familles \\
\hline Met 30 & NP, TD, vitré, rein & $\begin{array}{l}\text { Portugal, Japon, } \\
\text { États-Unis, Suède, } \\
\text { Turquie, Italie, France }\end{array}$ \\
\hline Ile 33 & NP, vitré & Issraël \\
\hline Leu 33 & NP, cœur & États-Unis \\
\hline Pro 36 & NP, vitré, cœur, rein & Grèce \\
\hline Thr 45 & Cœur & Portugal \\
\hline Arg 50 & NP & Japon \\
\hline His 58 & CC, cœur & États-Unis (Maryland) \\
\hline Ala 60 & Cœur, CC & États-Unis (Appalaches) \\
\hline Leu 64 & NP, cœur & États-Unis \\
\hline Tyr 77 & NP, vitré, rein & $\begin{array}{l}\text { États-Unis (IIlinois), } \\
\text { France }\end{array}$ \\
\hline $\begin{array}{l}\text { Ser } 84 \\
\text { Asn } 90\end{array}$ & $\begin{array}{l}\text { CC, cœur, vitré } \\
\text { NP, vitré }\end{array}$ & États-Unis (Indiana) \\
\hline Met 111 & Cœur & $\begin{array}{l}\text { Etats-Unis } \\
\text { Danemark }\end{array}$ \\
\hline Cys 114 & NP, vitré & J̦apon \\
\hline Ile 122 & Cœur & $\begin{array}{l}\text { États-Unis, Scandinavie, } \\
\text { Portugal }\end{array}$ \\
\hline
\end{tabular}

NP: neuropathie périphérique, TD: tube digestif, CC: canal carpien. 


\section{RÉFÉRENCES}

16. Jacobson DR, Gorevic PD, Buxbaum JN. A homozygous transthyretin variant associated with senile systemic amyloidosis evidence for a late onset disease of genetic etiology. Am J Hum Genet 1990 ; 47 127-36.

17. Jacobson DR, Reveille JD, Buxbaum JN. Frequency and genetic background of the position 122 ( $\mathrm{Val} \rightarrow$ Ile) transthyretin gene in the black population. Am J Hum Genet 1991 ; 49 : 192-8.

18. Pitkanen P, Westermark P, Cornwell GG III. Senile systemic amyloidosis. $A m$ Pathol 1984; 117 : 391-9.

19. Olson LJ, Gertz MA, William DE, et al. Senile cardiac amyloidosis with myocardial dysfunction. N Engl J Med 1987 ; 317 : 738-42.

20. Westermark P, Sletten K, Johansson B Cornwell GG III. Fibril in senile systemic amyloidosis is derived from normal transthyretin. Proc Natl Acad Sci USA $1990 ; 87$ 2483-5.

21. Blake CCF, Geisow MJ, Oatley SJ Rerat B, Rerat C. Structure of prealbumin : secondary, tertiary and quaternary interaction determined by Fourier refinement a 18 angström. J Mol Biol 1978 ; 121 339-56.

22. Hamilton JA, Steinrauf LK, Liepniks $\mathrm{J}$, et al. X-ray crystal structure of the Met 30 variant of human prealbumin (transthyretin). In: Natvig JB, Forre $\mathrm{O}$, Husby G, et al., eds. Amyloid and Amyloido sis. Dordrecht: Kluwer Academic Publishers, 1990 : 579-82.

23. Furuya $H$, Saraiva MJM, Gawinowicz MA, et al. Production of recombinan transthyretin with biological activities toward the understanding of the molecular basis of familial amyloidotic neuropathy (FAP). Biochemistry $1991 ; 30$ : 2415-21.

24. Spencer RGS, Halverson KJ, Auger M McDermott AE, Griffin RG, Lansbury PT. An unusual peptide conformation may precipitate amyloid formation in Alzheimer's disease : application of solid-state NMR to the determination of protein secondary structure. Biochemistry 1991; $30: 10382-7$.

25. Shimada K, Maeda S, Murakami T, et al. Transgenic mouse model of familial amyloidotic neuropathy. Mol Bio Med 1989 ; 6 : 333-43.

26. Yi S, Takahashi $K$, Naito $M$, et al. Systemic amyloidosis in transgenic mice carrying the human mutant transthyretin (Met 30) gene. Am J Pathol 1991; 138 : 403-12.

27. Van Allen MW, Frohlich JA, Davis $\mathrm{JR}$, et al. Inherited predisposition to generalized amyloidosis. Neurology $1969 ; 19$ : 10-25.

28. Ostertag B. Familiere amyloid Erkrangung. Z Menschl Verrbungs Konstit Lehre 1950 ; $30: 15-27$.

29. Nichols WC, Dwulet FE, Liepnieks J Benson MD. Variant apolipoprotein AI as a major constituent of human amyloid. Bio- mutation Met 30 a été décrite.

Chez les Portugais, la maladie débute habituellement tôt dans la vie, entre 20 et 30 ans, et son évolution est rapide. En revanche, dans certaines familles portugaises et dans d'autres populations comme en Suède, le début est beaucoup plus tardif, après 60 voire 70 ans $[11,12]$. Or ces malades ont la même mutation Met 30 , et les raisons de cette différence sont inconnues. On sait toutefois qu'il ne s'agit pas d'une expression tardive du gène puisque la protéine mutée est présente dans le plasma de patients jeunes, asymptomatiques mais appartenant à des familles à révélation tardive [11] ; il n'y a pas non plus de dosage génique puisque les rares malades homozygotes ont une maladie identique à celle des hétérozygotes, à l'exception de l'atteinte vitréenne qui, dans une famille suédoise, n'existe que chez les homozygotes [12].

D'autres résultats montrent que la présence d'une mutation dans le gène de la TTR n'est pas suffisante pour qu'une amylose survienne. En effet, des mutations ont été mises en évidence chez des sujets indemnes d'amylose, par isoélectrofocalisation de la protéine ou par séquençage après PCR des régions codantes du gène de la TTR, ces deux techniques étant choisies pour cribler de larges groupes d'individus [13, 14].

Enfin, il existe plusieurs mutations de la TTR qui modifient son activité fonctionnelle de fixation de la thyroxine sans entraîner d'amylose. La mutation Ala 109 a été bien étudiée : les sujets porteurs de la mutation dans cette famille sont cliniquement euthyroïdiens et ont simplement une anomalie biochimique caractérisée par un taux de thyroxine totale élevée, alors que leur thyroxine libre est normale. Les tests de fixation ont expliqué cette anomalie en montrant que l'affinité de la TTR Ala 109 pour la thyroxine était augmentée [15].

L'ensemble de ces résultats suggère donc qu'en plus de la mutation de la TTR d'autres facteurs génétiques ou liés à l'environnement sont impliqués dans l'expression de la maladie. Certains composants non fibrillaires de la substance amyloïde pourraient jouer un rôle physiopathologique. Les plus sérieux candidats sont le composant amyloïde $\mathrm{P}$ et les glycosaminoglycanes, qui sont tous deux intimement liés aux fibrilles amyloïdes [1]. Toutefois, aucune donnée à ce jour ne permet de faire jouer un rôle actif déterminant à ces molécules. Enfin, des fragments de TTR sont présents à côté de la TTR entière dans les fibrilles, et cette protéolyse semble avoir toujours lieu dans la même région de la molécule. Il n'est donc pas exclu qu'elle participe à la formation des fibrilles, mais elle pourrait tout aussi bien être un événement postérieur à la fibrilloformation.

La mutation n'est pas nécessaire à l'amyloïdogenèse

Plusieurs mutations de la TTR sont associées à une amylose cardiaque prédominante (Tableau III), dont la mutation Ile 122, qui est présente dans plusieurs familles où la maladie se révèle tardivement, dans la septième ou huitième décennie [16]. Comme pour toutes les maladies héréditaires à révélation très tardive, la nature héréditaire de l'affection n'est pas toujours évoquée, et les moyens de l'affirmer rarement réunis. La fréquence du gène muté Ile 122 a été évaluéc sur une petite population de race noire, où elle a été trouvée à une fréquence de $1,1 \%$, donc très élevée [17].

On ne sait cependant pas si tous les sujets porteurs de la mutation développeront une amylose cardiaque. Au sein d'une famille atteinte d'amylose de la TTR, il y a bien une ségrégation de la mutation avec la maladie, et les sujets sans mutation sont indemnes. En revanche, au cours du vieillissement, la TTR normale peut former de l'amylose. Cette entité est l'amylose sénile systémique (ASS), qui touche préférentiellement le cœur et qui est trouvée dans 10 à $30 \%$ des autopsies de sujets de plus de 80 ans [18]. Le diagnostic d'ASS est en effet rarement fait avant l'autopsie, à l'exception de quelques cas où le diagnostic clinique a été porté et la nature de l'amylose prouvée grâce à une étude en immunofluorescence avec des anticorps anti-TTR de biopsie endomyocardique [19]. Cette capacité de la TTR normale à former de l'amylose in vivo au cours du vieillissement est renforcée par des expériences in vitro qui ont montré que, 
dans certaines conditions, des fibrilles amyloïdes pouvaient être obtenues avec de la TTR normale [20]. Cette propriété est probablement en rapport avec sa structure $\beta$-plissée particulièrement extensive, car elle concerne l'ensemble de la protéine, ce qui n'est pas le cas de toutes les protéines amyloïdes connues. Il reste à déterminer pourquoi, in vivo, la TTR normale ne se dépose sous forme d'amylose que très tardivement dans la vie, quels sont les facteurs liés au vieillissement qui favorisent ce dépôt, et quelle est la fréquence des formes héréditaires parmi l'ensemble des amyloses cardiaques de la TTR à révélation tardive.

Vers la compréhension de l'amyloidogénicité de la TTR

Les mécanismes intimes qui conduisent à la formation des fibrilles amyloïdes restent mal connues. Pour les amyloses héréditaires, il est logique de penser qu'il existe des changements de la structure de la protéine amyloïde induits par la mutation, et qu'ils jouent un rôle physiopathologique majeur même si d'autres facteurs interviennent sûrement, comme nous l'avons vu, et modulent l'expression de la maladie. Les mutations connues sont réparties de façon homogène dans la TTR et ne modifient donc pas la même région de la protéine (figure 2). Il est toutefois possible qu'elles induisent des modifications plus subtiles de sa structure spatiale, difficiles à mettre en évidence. L'avenir est ici à l'analyse directe de la structure spatiale de la TTR par diffraction aux rayons X (voir $\mathrm{m} / \mathrm{s}$ $n^{\circ} 4$, vol. 7, p. 326). La TTR normale a été extensivement étudiée par cette technique, et sa structure est bien connue [21]. Pour l'étude des variants de la TTR, la première difficulté réside dans le fait que la majorité des malades étant hétérozygotes pour la mutation, ils possèdent les deux formes de la protéine, normale et mutée, et qu'il n'est pas possible d'obtenir, à partir de leur plasma, de grandes quantités de TTR mutée à l'état pur. A partir du plasma d'un malade homozygote pour la mutation Met 30, une quantité suffisante de TTR a été obtenue et son étude a montré, au voisinage du résidu Met 30 , un léger déplacement du feuillet $\beta$ et un élargissement du site de

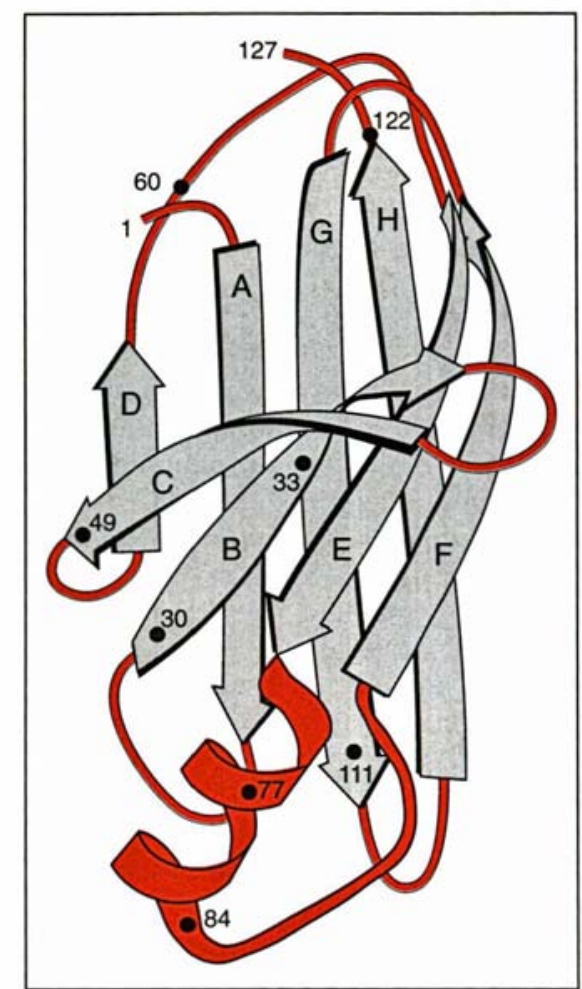

Figure 2. Structure secondaire du monomère de TTR. Les mutations sont réparties dans les huit feuillets $\beta$ plissés $(A$ à $H)$ de la TTR.

fixation de la thyroxine [22]. Cet obstacle est maintenant levé par le génie génétique, le gène de la TTR normale et de différents mutants ont été clonés dans un vecteur d'expression, et de grandes quantités de protéines recombinantes de bonne qualité ont été obtenues. Ces protéines ont été l'objet d'études fonctionnelles : capacité à former des tétramères et liaison à la thyroxine [23]. Ces travaux sont pour l'instant préliminaires, mais nul doute que l'étude de la structure tridimensionnelle des mutants de la TTR, par diffraction aux rayons $\mathrm{X}$, voire par résonance magnétique nucléaire en phase solide, comme cela a été récemment réalisé pour la protéine amyloïde $\beta$ de la maladie d'Alzheimer [24], apportera des éléments importants pour comprendre les changements induits par les mutations. Parallèlement à ces études in vitro, il était tentant de procéder à une approche in vivo de la maladie en créant des animaux transgéniques. Des souris $\mathrm{C} 57 \mathrm{BL} / 6$ ont ainsi été micro-injectées avec une construction faite du gène humain entier de la TTR Met 30 lié au promoteur murin de la méthallotionéine [25]. Les souris ont bien exprimé le transgène et la TTR mutée a pu être mesurée dans le sang. Les animaux ont été sacrifiés régulièrement tous les six mois jusqu'à 24 mois. En l'absence de signes cliniques, des dépôts d'amylose ont été mis en évidence dès le $6^{\mathrm{e}}$ mois et l'examen immunohistochimique a confirmé que l'amylose était bien composée de TTR [26]. Au $24^{\mathrm{e}}$ mois, de nombreux organes étaient infiltrés par l'amylose : particulièrement le cœur, le rein, la thyroïde et le tube digestif. Cette distribution tissulaire est comparable à celle observée à l'autopsic des malades décédés d'amylose héréditaire liée à la TTR. En revanche, l'amylose est totalement absente du système nerveux périphérique des souris transgéniques, alors que c'est chez l'homme l'organe le plus atteint. Cette discordance majeure n'est pas actuellement expliquée, mais plusieurs hypothèses peuvent être formulées pour expliquer cette absence de lésions des nerfs périphériques chez la souris : (1) le taux sérique de TTR Met 30 chez la souris transgénique, qui est de moitié inférieur au taux humain, ne serait pas assez élevé ; (2) la production de TTR Met 30 par les plexus choroïdes, qui n'existe pas chez la souris, serait indispensable ; (3) elle serait due à des caractéristiques anatomiques, histologiques ou métaboliques propres à la souris. Pour tester ces trois hypothèses, de nouvelles expériences devront créer : pour la première hypothèse, des souris exprimant plus fortement le transgène TTR Met 30 dans le sang; pour la deuxième, des souris exprimant le transgène dans les plexus choroïdes ; pour la troisième, d'autres espèces d'animaux transgéniques. Paradoxalement, cette discordance entre le modèle transgénique et l'homme permettra peut-être de mieux comprendre la spécificité tissulaire du dépôt amyloïde, qui est un des aspects les plus mystérieux et l'un des plus difficiles à aborder de la physiopathologie de l'amylose.

Ces animaux seront sans nul doute des modèles irremplaçables pour l'étude de la cinétique du dépôt amyloïde et devraient permettre de comprendre pourquoi cette maladie héréditaire se révèle tardivement; ils 


\section{RÉFÉRENCES}

30. Jones I.A, Harding JA, Cohen AS, Skinner M. New USA family has apoliprotcin Al (Arg 26) variant. In : Natvig JB, Forre O, Husby $\mathrm{G}$, et al., eds. Amyloid and Amyloidosis. Dordrecht : Kluwer Academic Publishers, 1990: 385-8.

31. Nichols WC, Gregg RE, Brewer HB, Benson MI). A mutation in apolipoprotein $\mathrm{AI}$ in the Iowa type of familial amyloidotic ncuropathy. Genomics $1989 ; 8: 318-23$.

32. Merctoja J. Familial systemic paraamyloidosis with lattice dystrophy of the cornca, progressive cranial neuropathy, skin changes and various internal symptoms (a previously unrecognized heritable syndrome). Ann Clin Res 1969; 1 : 314-24.

33. Maury CPJ, Alli K, Baumann M. Finnish hereditary amyloidosis amino acid sequence homology between the amyloid libril protein and human plasma gelsolin. Febs Letl 1990 ; 260 : 85-7.

34. Levy E, Haltia M, Fernandez-Madrid $\mathrm{I}$, et al. Mutation in gelsolin yene in Finnish hereditary amyloidosis. I Exp Med $1990 ; 172: 1865-7$

35. Hiltunen ' $\mathrm{T}$, Kiuru $\mathrm{S}$, Hongell $\mathrm{V}$, Heliö T, Palo J, Peltonen I. Finish type of familial amyloidosis : cosegregation of $\mathrm{Asp}_{187} \rightarrow$ Asn mutation with the discase in threc large families. Am / Hum Genet 1991 ; 49 : 522-8. 36. Gorevic PI), Muno\% PC, Gorgone G, et al. Amyloidosis duc to a mutation of the gelsolin gene in an American family with lattice corncal dystrophy type II. N Engl I Med 1991; 325: 1780-5.

37. Hida ' $\mathrm{T}$, Tsubota K, Kigasawa K. Clinical features of a newly type of lattice corneal dystrophy. Am .J Ophtalmol $1987 ; 104$ : 241-8.

38. Haltia M, Ghiso J, Wisniewski 'T, Kiuru S, Miller 1), Frangione B. Gelsolin variant and $\beta$-amyloid co-occur in a case of Alzheimer's with I,ewy bodies. Neurobiol Aging 1991; 12 : 313-6

39. Wisnicwski $\mathrm{I}$, Haltia $\mathrm{M}$, Ghiso J, Frangione B. Lewy bodies are immunoreactive with antibodies raised to gelsolin related amyloid-Finish type. Am .J Pathol 1991 : 5: 1077-8.3

40. Rajagopalan K, Tay Ch. Familial lichen amyloidosis. Report of 19 cases in 4 generations of a Chinese family in Malaysia. $\mathrm{Br}$ Dermatol 1972 ; 87 : 123-9.

41. Koussef BG, Espinoza C, Zamore GA. Sipple syndrome with lichen amyloidosis as a paracrinopathy : pleiotropy, heterogencity, or a contiguous gene? I Am Acad Dermatol $1991 ; 25$ : 657-61.

42. Partington MW, Prentice RSA. Xlinked cutancous amyloidosis : further clinical and pathological obscrvations. $\mathrm{Am}$.J Med Genet $1989 ; 32: 115-9$

43. Goren H, Steinbere MC, Farboody GH. Familial oculoleptomingeal amyloidosis. Brain 1980; 103: 473-95.

44. Ushiyama $M$, Ikeda $S$, Yanagisawa $N$. 'Iransthyretin-type cercbral amyloid angiopathy in type I familial amyloid polyncuropathy. Acta Neuropathol 1991; 81: 524-8.

45. Benedik\% F, Blöndal $\mathrm{H}$, Gudmunsson G. Skin deposits in hereditary cystatin C amyloidosis. Virchows Arch A Pathol Anat $1990 ; 417: 325-31$ pourront aussi être utilisćs comme outil pharmacologique pour tester des molćcules visant à empêcher la formation des fibrilles.

\section{Les autres amyloses héréditaires}

Les amyloses de l'apolipoprotéine A1

L'apolipoprotćine A1 est la protéine amyloïde présente chez deux familles ayant des maladies cliniquement très différentes. La première famille a une amylose dont l'aspect clinique est très proche de celui des amyloses de la TTR, et seule une fréquence accrue d'ulcc̀res gastriques avait conduit à son individualisation clinique [27]. La seconde famille a une amylose rénale et hépatique, et appartient à un petit groupe d'amyloses familiales dont le modc̀le a été décrit initialement par Ostertag [28]. Cette varićtć n'affecte pas le système nerveux pćriphćrique, atteint préférentiellement lc rein, mais les signes cliniques ne sont pas compatibles avec le diagnostic de maladie périodique ou de syndrome de Muckle et Wells défini par l'association d'arthrites, d'une urticaire, d'une surdité de perception, et parfois d'unc amylose AA (Tableau I) [3]. La protéine fibrillaire est, dans ces deux familles, un fragment de l'apolipoprotéinc A1 $[29,30]$. Très curicusement, la mutation est la même pour les deux familles, alors que la distribution tissulaire des lésions y est très différente. Ce fragment protéique contient une substitution : une arginine remplace une glycine en position 26 et correspond à unc mutation ponctuclle qui a été confirméc par sćquençage de l'ADN $[30,31]$.

L'amylose de la gelsoline

Décrite initialement en Finlande, où plusieurs familles sont touchées par la maladic, puis de façon isolćc dans d'autres pays, cette amylose familiale de transmission autosomique dominante est caractérisćc essentiellement par la double association d'une neuropathie des nerfs crâniens et d'une atteinte cornéenne appeléc dystrophie grillagće [32]. Chez des malades finlandais, la protéine qui a été purificee à partir des fibrilles est un fragment de la gelsoline, une protéine dont il existe deux isoformes : l'une appartient au cytosquelette et se lic à l'actinc, l'autre est circulante. Cette gelsoline est anormale et possède une substitution de l'acide aminé en position 187 où un acide aspartique est remplacé par une asparagine [33]. Cette substitution est secondaire à une mutation adćnine-guanine au nucléotide 654 [34]. La preuve définitive de la responsabilité de cette mutation dans la maladic a ćté apportćc par l'ćtude de trois grandes familles finlandaises dans lesquelles la mutation sćgrège bien avec la maladie [35]. La même mutation a été mise en ćvidence chez un malade américain [36]. Il existc, par ailleurs, plusicurs formes d'amyloses hérćditaires cornćennes isolées, dont on ne sait rien de la nature biochimique [37].

Des résultats préliminaires récents suggèrent que la gelsoline pourrait intervenir dans la physiopathologie de lćsions du système nerveux central n'ayant pas de rapport avec l'amylose : les corps de Lewy. Ces structures sont caractéristiques de la maladic de Parkinson quand elles sic̀gent dans les noyaux gris centraux, mais peuvent être plus diffuses dans le cerveau et l'expression clinique de la maladic est alors une démence. Or, le malade originel atteint d'une amylose de la gelsoline était dément et l'examen histologique de son cerveau a montré des corps de Lewy diffus qui reconnaissent des anticorps dirigés contre le fragment de gelsoline extrait de la substance amyloïde [38]. La même fixation a été obscrvéc sur des corps de Lewy de malades atteints de maladie de Parkinson [39]. Autres variétés d'amylose

Dans plusieurs varićtés d'amylose héréditaire, la protéine amyloïde n'a pas été définitivement caractérisće, et la mutation est inconnue. On trouve dans ce groupe: (1) les familles attcintes d'amylose cutanée de type lichenoïde, où, comme dans les formes sporadiques du même type, la protéine amyloïde pourrait être une kératine [40] ; (2) des familles atteintes de nćoplasie endocrinienne multiple de type II a (NEM II $\mathrm{a}$ ), où certains malades ont de discrètes lésions d'amylose cutanée, dont l'examen immunohistochimique suggère que la protéine amyloïde est là cncore une kćratine. Ces dépôts d'amylose cutanée sont donc d'une nature biochimique différente de ceux trouvés 
dans le stroma du carcinome médullaire de la thyroïde, une des tumeurs caractéristiques de la NEM II a, qui sont, quant à eux, formés de calcitonine [41] ; (3) la famille décrite par Partington, où les garçons ont de discrètes lésions d'amylose cutanée ct une maladie polyviscérale grave, sans rapport avec l'amylose, alors que les filles ont seulement des lésions cutanées [42] ; (4) des formes oculoméningées [43].

\section{Amyloses cérébrales}

La distinction que nous avons établic entre les amyloses du système nerveux central et les autres est un peu artificielle car : (1) dans les amyloses de la TTR, des dépôts amyloïdes sont présents également dans les méninges et les vaisseaux cérébraux [44] ; (2) dans l'hémorragic cérébrale héréditaire de type islandais, où l'amylose cérébro-vasculaire est forméc de cystatine C, la biopsie cutanéc permet un diagnostic précoce de la maladie, et de nombreux viscères sont envahis à l'autopsic [45]. Les amyloses de la TTR ont de nombreux points communs avec la maladie d'Alzheimer. En effet, ce sont deux maladies où le dépôt amyloïde est le processus pathogène initial, et dont il existe, d'une part, des formes héréditaires où les mutations siègent dans la protéine amyloïde et, d'autre part, des formes non héréditaires. Les travaux à venir devront porter sur la compréhension du rôle joué par les mutations et par les facteurs associés au vicillissement dans la physiopathologie de ces maladies. A priori, la TTR est unc molécule plus facile à étudier que le précurseur de la protéine amyloïde $\beta$ (APP) car elle est produite par le foic, circule dans le plasma, et la protéolyse dont elle fait l'objet avant son dépôt est nulle ou limitée, alors que la cellule qui synthétise l'APP est inconnue, l'APP est transmembranaire et ne circule pas, et elle subit une protéolyse complexe avant son dépôt.

Ainsi, les amyloses héréditaires de la TTR, qui sont des maladies rares puisque la prévalence des différents variants est estiméc à 1 sur 100000 aux États-Unis, pourraient contribuer à la compréhension d'une maladie fréquente : la maladie d'Alzheimer, à moins que, compte tenu de l'énorme effort de recherche entrepris dans le domaine de la maladie d'Alzheimer, ce ne soit le contraire qui se produise...

\section{Summary}

Hereditary amyloidoses

Amyloidosis is a common final pathway for protein deposition in tissues. Several amyloidoses are hereditary disorders with clinical and genetic heterogeneity. In these diseases, there is a mutation in the gene coding for the corresponding amyloid protein : apolipoprotein A1, gelsolin or most frequently transthyretin (TTR). In the discase secondary to TTR amyloid deposits, several major organs are involved : peripheral nerves, heart, kidney, eye, digestive tract, and its course is scvere. About fifteen mutations have been found in the TTR gene, with various clinical patterns. DNA and protein technologies now allow the diagnosis of the asymptomatic carricrs, and the prenatal diagnosis in the severe forms of the disease. Further in vitro studies using biophysical procedures such as X-ray cristallography, will teach us how the mutation is responsible for increased amyloidogenicity. Transgenic mice with mutated TTR are a good model of the disease. They should address the question of kinetics and tissue specificity of the amyloid deposits and will allow pharmacologic intervention.
TIRÉS A PART

G. Grateau . 